\title{
THE DETERMINATION OF IMPURITIES IN COMMERCIAL COPPER.
}

\section{By Bertram Blount.}

THE method given by Professor Hampe (Chem. Zeit., 1893, xvi, 1691, see abstract on next page) depending upon the precipitation of the copper as cuprous thiocyanate and the determination of the metals present as impurities in the filtrate, has been in constant use in my laboratory for some years past, having been devised by me without knowledge of its previous employment.

The principle of the process is identical with that adopted by Professor Hampe, but there are a few differences in detail which add somewhat to its convenience. In most cases 10 grammes of the copper to be analysed is sufficient to yield ponderable quantities of the impurities to be determined, although of course this quantity may be increased if necessary. In fact, the great merit of the method consists in the fact that large quantities of material may be used without the trouble of handling unwieldy precipitates, as when once the copper has been precipitated as thiocyanate nothing further has to be done to it in the way of manipulation. When first working the process, I was accustomed to use 10 grammes of the copper and wash the resulting thiocyanate. This plan was speedily abandoned in favour of that mentioned by Professor Hampe, of drawing off a definite portion of the supernatant liquor and allowing for the volume occupied by the precipitate. In order to do this, it was 
necessary to ascertain the specific gravity of cuprous thiocyanate. This constant was not given in any book which I consulted, and accordingly was determined on a sample prepared in the course of one of the previous analyses. The figure thus found for the specific gravity of cuprous thiocyanate at $15^{\circ} \mathrm{C}$. compared with water at the same temperature, was 2.846 , somewhat lower than that found by Professor Hampe $(2 \cdot 999)$. The reason for this discrepancy may be that the precipitation was conducted under different conditions which had an influence on the density of the salt. Taking the figure for the specific gravity of cuprous thiocyanate given above, a simple calculation showed that if 13.215 grammes of copper were taken, dissolved, precipitated as hiocyanate, and the solution together with the precipitate made up to 1 litre, on drawing off 750 c.c. the impurities corresponding to 10 grammes of copper would be obtained. By using this plan, all need for calculation of the results of the analysis is avoided.

Another point in which my process differs from that of Professor Hampe is that the copper is dissolved in aqua regia instead of nitric and sulphuric acids. By the use of this solvent, and the subsequent removal of the excess of nitric acid, I obtain a clear solution with all ordinary samples of copper, and avoid the necessity for making a separate analysis of the insoluble residue, a result which is, I venture to think, a distinct advantage. Thus all the common impurities of commercial copper, with the exception of gold, silver, sulphur, and oxygen, can be determined in one and the same solution, in the absence, moreover, of all but traces of copper, making the analysis a vastly simpler task than by the older methods.

There is only one other matter that deserves mention. Although arsenic can be determined in the solution from the cuprous thiocyanate, I have found it more expeditious, easy, and accurate to estimate it on a separate portion by distillation with ferric chloride and hydrochloric acid, as it is thus finally obtained as pure arsenious sulphide, and can be conveniently weighed in this form. There is only one drawback to this process, and that is the difficulty of obtaining reagents free from arsenic, making it necessary for the chemist to prepare them himself.

Broadway, Wrstminster, S.W.

The Determination of Foreign Metals in Commercial Copper. W. Hampe. (Chem. Zeit., 1893, xvii. 1691-1692.)-The following is the method adopted by the author: 25 grammes of the sample are dissolved in a mixture of 200 c.c. of water, 100 c.c. of pure concentrated sulphuric acid, and $45-46$ c.c. of nitric acid of sp. gr. 1.21. The quantity of the last-named reagent is reckoned so as to afford a small excess over that necessary for the oxidation of the quantity of copper taken, while the amount of sulphuric acid represents a considerable surplus, in order to prevent the separation of basic salts of bismuth and antimony when the solution is subsequently diluted. When the whole of the copper is dissolved, the solution is diluted with 200 c.c. of water to prevent the formation of crystals of copper sulphate. The resulting liquid is generally clear, but it may be turbid from the separation of in. soluble antimoniates of copper and bismuth, which must in that case be filtered off and examined separately. The original solution, or the clear filtrate, as the case may 
be, is warmed to $40^{\circ} \mathrm{C}$, and treated with sulphur dioxide in a rapid stream to decompose the remainder of the nitric acid, the reduction being complete in about half an hour provided the temperature specifed, which is the most favourable for the reaction, be observed. The solution, which should smell of sulphur dioxide, may be turbid from the presence of metallic silver precipitated by the reducing agent. Should it be desired to determine the silver in the wet way, the precipitation of traces not reduced by the sulphur dioxide is effected by the addition of a few drops of hydrochloric acid and the mixed precipitate of silver and silver chloride filtered off, converted completely into chloride and weighed in the usual manner. If, on the other hand, a dry assay for silver is to be made, the turbidity due to the separation of metallic silver may be disregarded, and the main body of liquid, together with the trace of metallic silver, is transfixed to a two-litre flask and precipitated with pure potassium thiocyanate, a rapid stream of sulphur dioxide being meanwhile maintained. A slight deficiency of potassium thiocyanate is used, so that a small fraction of the copper may remain in solution. The solution of potassium thiocyanate is of such strength that about .500 c.c. are required to precipitate 25 grammes of copper. The total bulk is then made up to two litres, the precipitate allowed to subside and a known volume of the supernatant liquor filtered off. 1800 c.c. is a convenient amount to take. The excess of sulphur dioxide is driven off by evaporation, and the foreign metals originally present in the copper, such as antimony, arsenic, bismuth, tin, iron and nickel, are separated and determined by the customary analytical methods. In making the calculations of the analysis it is necessary to correct for the volume of the cuprous thiocyanate in order to ascertain with what fraction of the 25 grammes of copper originally taken the quantity of liquid drawn off after precipitation corresponds. This involves a knowledge of the specific gravity of cuprous thiocyanate. The author has determined this value, and finds it to be 2.999 , so that the volume occupied by the cuprous thiocyanate from 25 grammes of copper is 15.98 c.c. The total bulk of liquid in the two-litre flask may therefore be taken as 1984 c.c., and the relation between this number and that of the liquid drawn off, viz., 1800 c.c., determines upon what fraction of the 25 grammes of copper the estimation of foreign metals has been effected. Test analyses of pure copper, to the solution of which known quantities of impurities had been added, prove the accuracy of the method.

B. B.

The Separation and Volumetric Estimation of Lead. Lindeman and Motteu. (Bull. Soc. Chim., 1893, x. 812, through Chem. Zeit.)-The authors have found that chloride of lime is capable of acting on native sulphides, converting their metals into oxides or peroxides and their sulphur into sulphate. The oxidation appears to be complete, and thus can be utilised for the estimation both of the metal and sulphur. In the case of galena the lead is obtained as peroxide, and can be estimated iodometrically. The analysis is performed by rubbing down 0.5 to 1.0 gramme of the sample in an agate mortar with a few drops of a solution of chloride of lime, and finally triturating the product with 30 to 40 c.c. of the same reagent. A little hydrochloric acid is then added, and the whole warmed until chlorine ceases to be evolved. The solution is diluted, and the lead peroxide washed by decantation, bringing as little as possible upon the filter, and treated with potassium iodide and hydrochloric acid; the liberated iodine is titrated with hyposulphite. The presence 
of either iron or copper interferes, as each is capable of liberating iodine and is therefore reckoned as lead. When iron alone is present it is sufficient to precipitate the lead as sulphide with sulphuretted hydrogen in acid solution, and treat the sulphide as if it were the original ore. When copper has to be removed, the sulphide is precipitated as described above, and washed with potassium cyanide, which dissolves the cupric sulphide and leaves the lead sulphide to be dealt with as before.

B. B.

The Determination of Phosphoric Acid by the Titration of the Yellow Precipitate with Standard Alkali. (Jour. Franklin Inst., 1893, cxxxvi., 362-376.) -The author shows that 23 molecules of sodium carbonate are required to neutralize the molybdic acid in every molecule of the yellow precipitate when the composition of this is $6 \mathrm{NH}_{4} \cdot \mathrm{P}_{2} \mathrm{O}_{8} \cdot 24 \mathrm{MoO}_{3}$. The best standard alkali for the purpose is a solution of caustic potash, of which 1 c.c. is equivalent to 1 milligramme of $\mathrm{P}_{2} \mathrm{O}_{5}$ in the yellow precipitate. Such a solution neutralizes normal acid in the proportion of $100: 32 \cdot 65$ volumes, and may be made from potash which has been freed from carbonate by means of baryta-water, on this basis; or it may be standardized by titration of the yellow precipitate obtained from pure sodium phosphate, treated in the manner described below.

The ammonium molybdate solution is prepared by dissolving 90 grammes of the salt in somewhat less than a litre of water, allowing the solution to settle, and decanting the clear liquor into a litre flask. The small quantity of insoluble molybdic acid always present is dissolved in a little ammonia, and added to the main solution. If the molybdate contains a trace of $\mathrm{P}_{2} \mathrm{O}_{5}$, a few decigrammes of magnesium sulphate, and ammonia to faint alkalinity, are added. The whole is then made up to one litre. Each c.c. precipitates 3 milligrammes of $\mathrm{P}_{2} \mathrm{O}_{5}$.

The standard acid must be equivalent to the alkali, and may be made by adding 326.5 c.c. of normal acid to one litre.

The analysis is conducted as follows: One gramme of phosphate rock, or 2 or 3 grammes of fertilizer, are dissolved in nitric acid, and, without evaporating to dryness, diluted to 250 c.c. The solution need not be filtered, inasmuch as the volume of the insoluble matter seldom amounts to 0.05 c.c. Twenty-five c.c. of the solution are delivered into a four-ounce beaker and neutralized with ammonia - until a precipitate just begins to form-and then treated with 5 c.c. of nitric acid of $1 \cdot 4$ sp. gr. Ten c.c. of a saturated solution of ammonium nitrate are added, and the solution diluted to a volume of 50-75 c.c. It is then brought to a full boil, removed from the lamp, and 5 c.c. of the aqueous solution of ammonium molybdate added. This is followed by a second and a third 5 c.c. if necessary, the precipitate is allowed to settle, and filtered at once. It is washed thoroughly by decantation and on the filter. The filter and precipitate are transferred bodily to the beaker. Standard alkali is then run in, and at least 0.5 c.c. of a phenolphthalein solution (1 per cent.) added, then standard acid until the colour vanishes. Each c.c. of alkali equals one milligramme of $\mathrm{P}_{2} \mathrm{O}_{5}$.

A. G. B. 